\title{
Human Body Analysis and Diet Recommendation System using Machine Learning Techniques
}

\author{
M. Geetha ${ }^{1}$, C. Saravanakumar ${ }^{2}$, K. Ravikumar ${ }^{3}$, and V. Muthulakshmi \\ \{geetham1@srmist.edu.in ${ }^{1}$, mailofcsk@gmail.com ${ }^{2}$ \\ ravikumarcsephd@gmail.com ${ }^{3}$ and hoditlabaffairs@stjosephs.ac.in $\left.{ }^{4}\right\}$ \\ Professor, Department of Computer Science and Engineering, School of Computing, College of \\ Engineering and Technology, Faculty of Engineering and Technology, SRM Institute of Science and \\ Technology, Vadapalani, Chennai, India ${ }^{1}$ \\ Associate Professor, Department of Information Technology, \\ St.Joseph's Institute of Technology, OMR, Chennai - 600119, India ${ }^{2}$ \\ Associate Professor, Department of Computer Science and Engineering, \\ Rrase College of Engineering, Chennai - 600119, India ${ }^{3}$ \\ Associate Professor, Department of Information Technology \\ St. Joseph's College of Engineering, OMR, Chennai 600 119, India ${ }^{4}$
}

\begin{abstract}
Nowadays the human faces the problem in maintaining the health condition in proper level. The problem is occurred due to excess consumption of the food which leads to obesity and also causes health issues. Mismanagement of the human health system is monitored using automated system which provides the report to the person. Traditional health monitoring model lacks in the accuracy of the report so it is improved by implementing intelligent diet control system. This provides the human choose and consumed proper food based on their health condition and extend the life time. The main objective of the proposed system analyses the body and provides the diet report to the person accurately. Exiting techniques are only support the person based on the current activity which leads the reliability problem. The proposed model uses the machine learning approach which analyzes the body of the human with pre medical history and predict the future health condition over the year. It provides the diet recommendation systems by considering the current and past food consumption record and recommend proper diet report with more reliable manner.
\end{abstract}

Keywords: Machine Learning, Deep Learning, Recommendation Systems, Healthcare, Data Mining

\section{Introduction}

Machine learning is a process to predict the future without any intervention of the human and any program. It is pervasive computing paradigm which applied over various areas such as speech processing, searching of a web, automated vehicles and so on. It supports the human life by solving new problems with vast amount of data with more accurate manner. It is evolved from data a mining technique which uses the heterogeneous data from various sources. AI (Artificial Intelligence) is a broad area which provides the ability like human through computer and related programs in order to solve the problems. Machine learning algorithms follow AI approach for improving the training ability of the models. There are 
three different methods of doing the machine learning algorithms namely supervised, reinforcement and unsupervised algorithms. Supervised algorithms uses the labeled data and known data. Reinforcement algorithms are interacts with the environment and performs learning through agents with two parameters reward and penalty. Unsupervised algorithm uses different data without any labeling and format. Classification and regression problems are solved by the supervised method in single class and multiclass category. Clustering and association based problems are solved by using unsupervised algorithms by discovering patterns in multiclass manner. Some mix up of labeled and un labeled data are handled by using semi supervised learning algorithms. Business always depends on the recommendation model to improve the profit in a successful direction. Recommendation algorithms uses large volume of data which are provided by the human based on the experience through feedback and some other methods. It filters out the suitable data from large data set and gives the high quality outcome to the customer. The reasons for the recommend systems are user interest selection, right delivery of the product to the user, discover the product with all categories; generate the content and user retention by applying high quality result. Various types of recommendation systems are exits they are popularity based, classification based, content based and collaborative based. Collaborative based is again divided into user based, item based and decomposition level. These types provide high quality searching result with efficient manner. Management of weight follows various techniques and process in the human life for living in long term. It depends on the healthy eating of food and routine physical activity by the human. This process always monitoring the human activity and track their actions in all time. Various factors are involved to for this process namely dietary control, human activity, disease and so on. Balancing of energy is plays vital role which is calculated by taking the difference over the total number of calories consumption by the person and calories burned by the person at the particular time. Traditional statistical analysis method uses pre and post study which is not detected effect in the obesity. The method with visualization tools with parameters provides the pattern to be followed by the human. Two types of analysis carried out namely with and without intervention effects [1].Diet elements related to demographic situation leads the gastric cancer. Machine learning algorithms uses the parameters which are help to the lifestyle of the human without disease. Classification algorithms are uses to control the false positive rate of the patient [2]. Human eating analysis is monitored needs the interaction to control the entire system. Wearable devices always provide the information continuously by considering food consumption level, speed of the eating and quantity of the food. The proposed monitoring system analyzes the dietary related to the captured images [3]. Correct food gives the healthiest life to the human and also categorized consumed food and not consumed food. Supervised based learning method classifies these food items from large collection of items [4]. The new learning method which is based on kernel method needs to collect related information. Genetic programming approach learns the suitable features through SVM approach [5]. Dietary monitoring system suffers the threats like diabetes, obesity and so on. This system collects the feedback from various human and provides the accurate information with wearable elements [6]. The existing methods are analyzed the diet system with various dimensions which suffers the intelligent support and also lacks the accuracy. This problem is overcome by using recommendation system with machine learning approach with high level accuracy. This model provides the information to the individual to follow their own dietary report without consulting any dietician. 


\section{Proposed System}

Human data are collected from various data sources with different data format and representation which is used for further human obesity analysis. These data are stored into database for persistent storage with large collection of raw data. The features are analyzed and extracted from the stored data. The hyper features are selected and preprocessed with suitable analysis. BMI index of the every human is calculated based on the height and weight. It identified the suitable level which provides the accurate information for diet maintenance. Calories range of the human is calculated from the BMI which is calculated from extracted features. Pre medical history also considered for precise evaluation. These parameters are fitted into the machine learning model which predicts the diet plan of the human in order to maintain the proper health in longer time. The model is evaluated with various performance analyses and improves the accuracy. It also gives the recommendation report to the human for better maintenance of their body. The features are entered by the user such as height, weight, gender type, age of the human and regular exercise practice etc. This recommendation system needs the age and BMI feature with appropriate level. The main objective of the proposed model is to maintain good health without any disease to the human. BMR (Basal Metabolic Rate) is the total number of calories needed for maintaining proper functioning of the human body. It is an energy amount which is extended related to the digestive system. It is going to reach inactive condition to 12 hours. The BMR rate of the men and woman related to the parameters like weight, height and age of the human. The calories are estimated through the consideration of the BMR rate. This estimation process done through different formula with different parameter combination, so it is selected based on the accuracy level. The complete analysis provides the diet plan which includes the food to be taken by the human per day. It also provides the schedule to the human for doing regular activity. Figure 1 shows that the architectural model of the proposed algorithm.

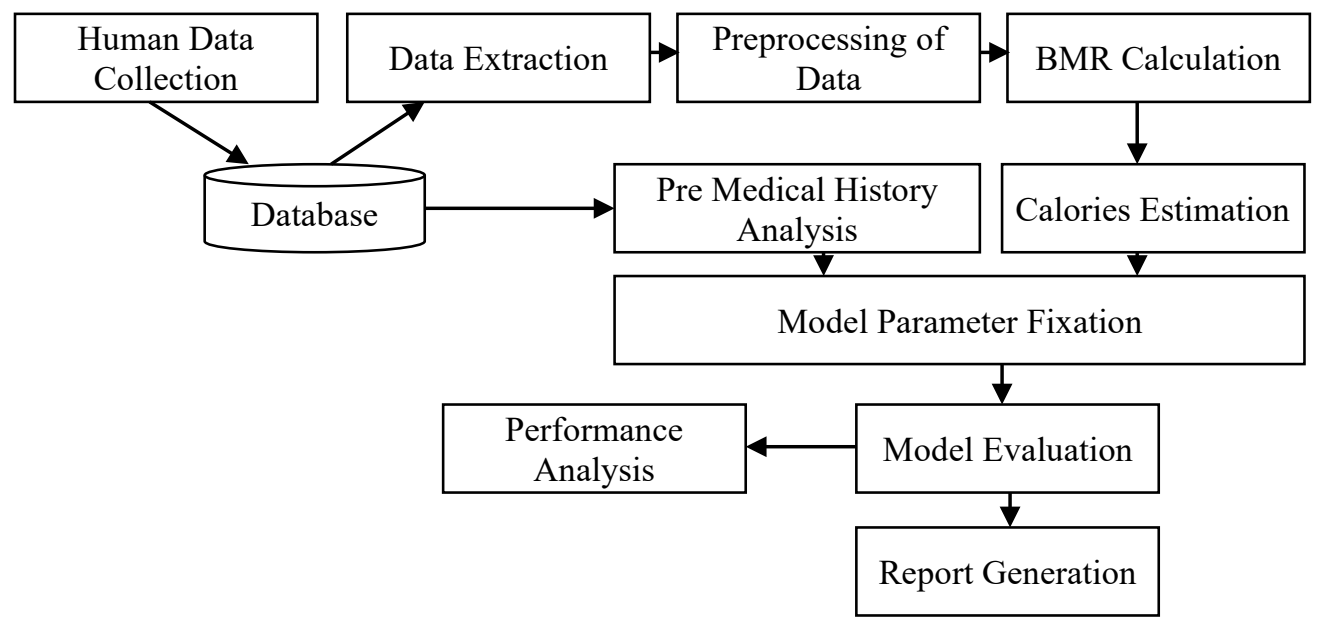

Fig. 1. Architecture of the proposed model 


\section{Algorithm}

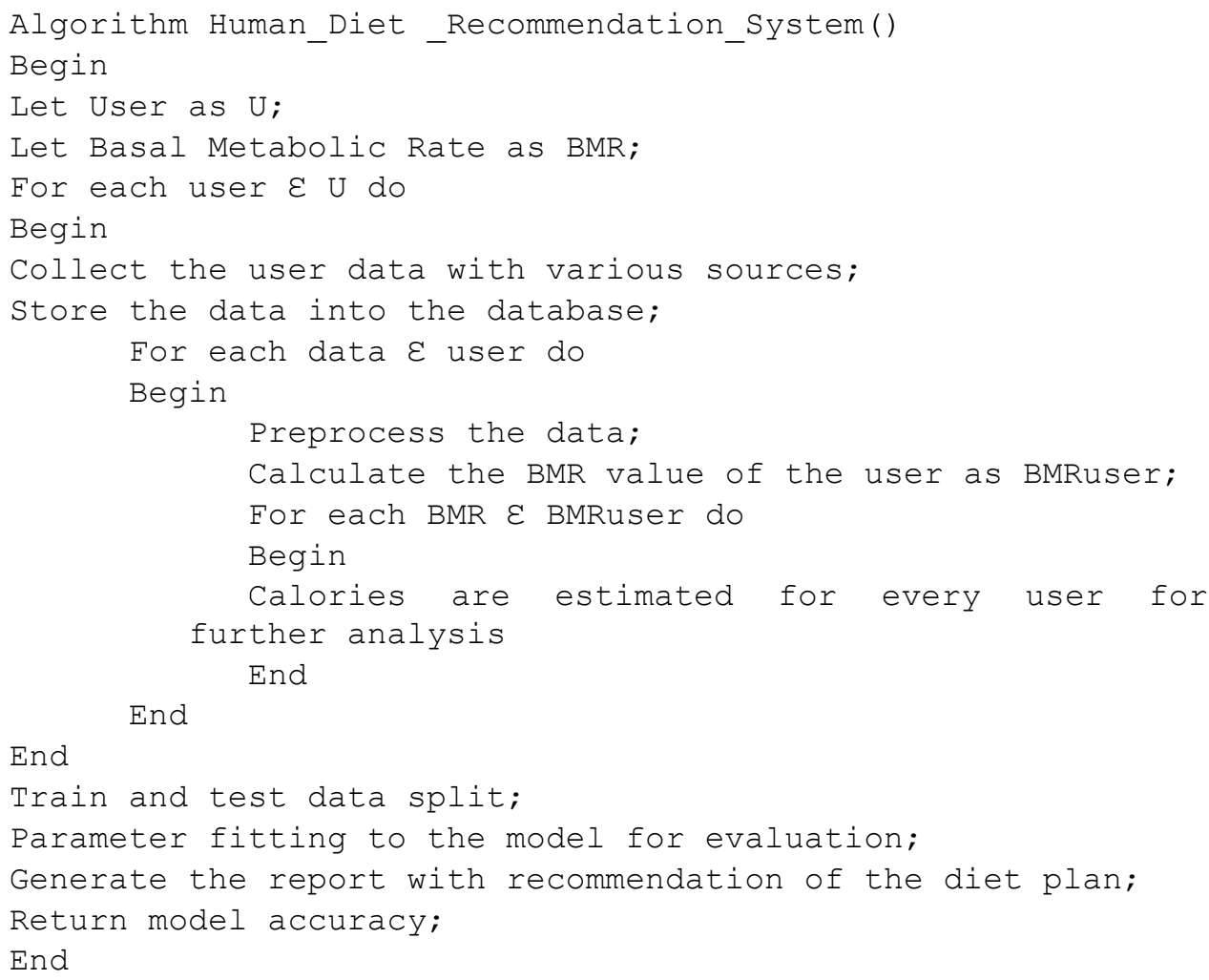

\section{Analysis of Recommendation Systems}

Recommendation system provides exact data which is very relevant to the customer for purchasing the product in online mode. This type of the system also applied to the educational domain for exploring the unexplored data. It also gives the support to the student for getting the knowledge in all field of study. APARELL method provides the guidelines to the students for selecting suitable content with ontology search [7]. Context based recommender system (CARS) uses two phases process with multidimensional manner. Video based system solves the challenges such as approximation and attributes of context. It applies the rules with minimum roughness while handling the attributes during selection [8]. The services and products are selected by the user and taking decision needs the intelligent support via precise recommendation model. Various approaches are used for extraction purpose with the features like content oriented, integrated approach, filtering method and federation approach. Multi agent model provides high security by protecting the code related problems. Objected oriented approach applies the high level of protection during the user interaction [9]. In tourism domain uses advanced devices and techniques for providing recommendation to the customer through mobile and wireless devices. It supports to attain the target optimally by considering the parameters like preferences of user, context of environment and customer. The data are 
maintained in the centralized repository in order to achieve high quality targets [10]. Trust oriented recommendation systems (TARS) are used to achieve high efficiency related to the accuracy. It also uses multi dimensional recommendations to the users [11]. Table 1 represents the analysis of the statistical parameters in the proposed model. Figure 2 and figure 3 provides the analysis of the food consumption of correct and incorrect respectively. Analysis of the recommendation accuracy is shown in figure 4 .

Table 1.Statistical Analysis

\begin{tabular}{lccc}
\hline \multicolumn{1}{c}{ Statistical Parameters } & Estimated Obesity & Calories Consumption & Calories Burned \\
\hline Mean & 0.299506 & 0.519506 & 0.276506 \\
Standard Deviation & 0.113258 & 0.113258 & 0.113258 \\
Min & 0.070353 & 0.290353 & 0.047353 \\
$\mathbf{2 5 \%}$ & 0.240699 & 0.460699 & 0.217699 \\
$\mathbf{5 0 \%}$ & 0.282818 & 0.502818 & 0.259818 \\
$\mathbf{7 5 \%}$ & 0.313238 & 0.533238 & 0.290238 \\
Max & 0.922932 & 1.142932 & 0.899932 \\
\hline
\end{tabular}

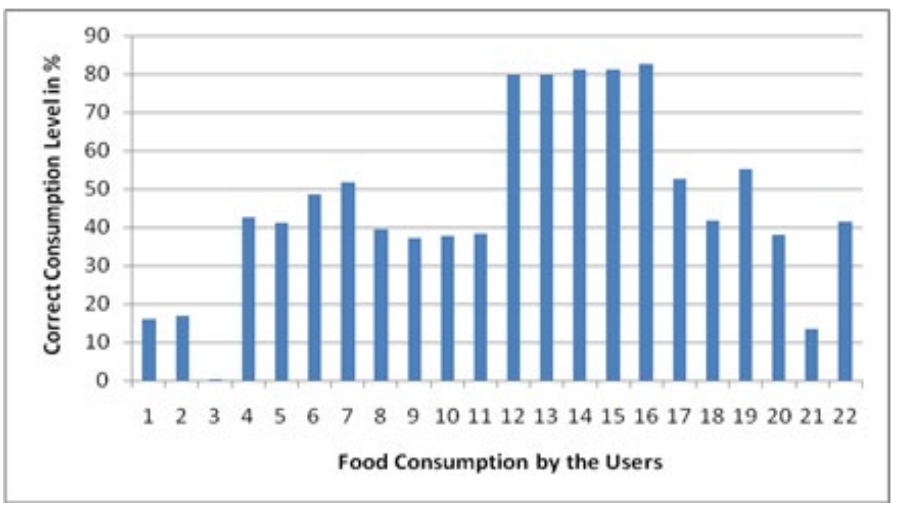

Fig. 2.Correct food analysis

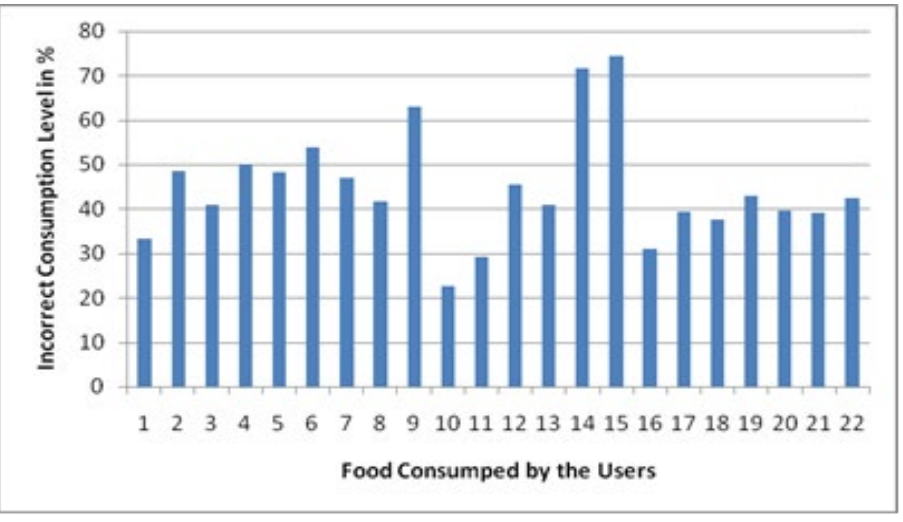


Fig. 3.Incorrect food analysis

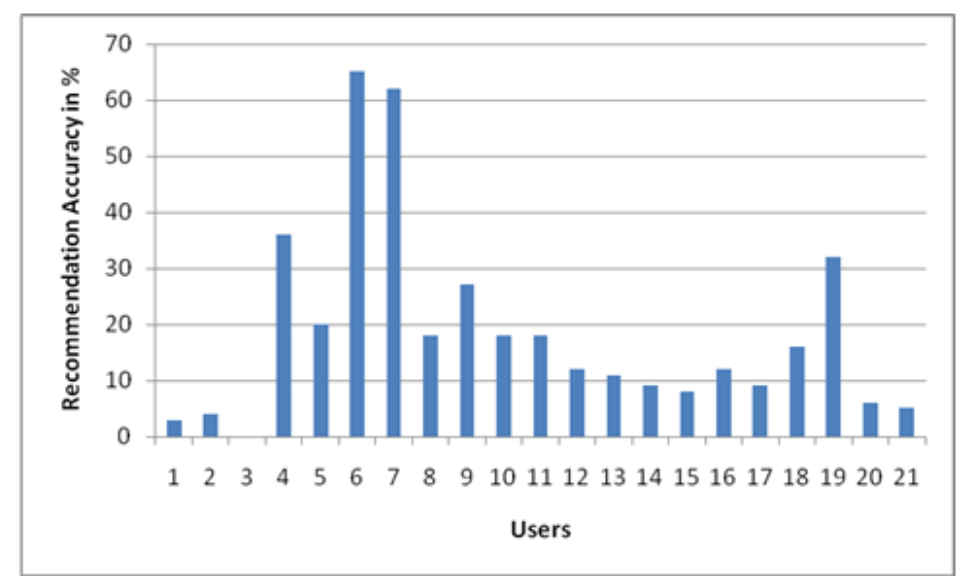

Fig. 4.Accuracy analysis

\section{Results and Analysis}

Kidney disease is identified and provides the suitable diet plan to the patients using machine learning approach. It also considers the pre medical records of the patients. Various multi class machine algorithm models are compared and selected the random forest provides maximum accuracy [12]. Random based machine learning method with conditions applied for various diseases. It achieves better accuracy with two parameters such as structural based features and data [13].

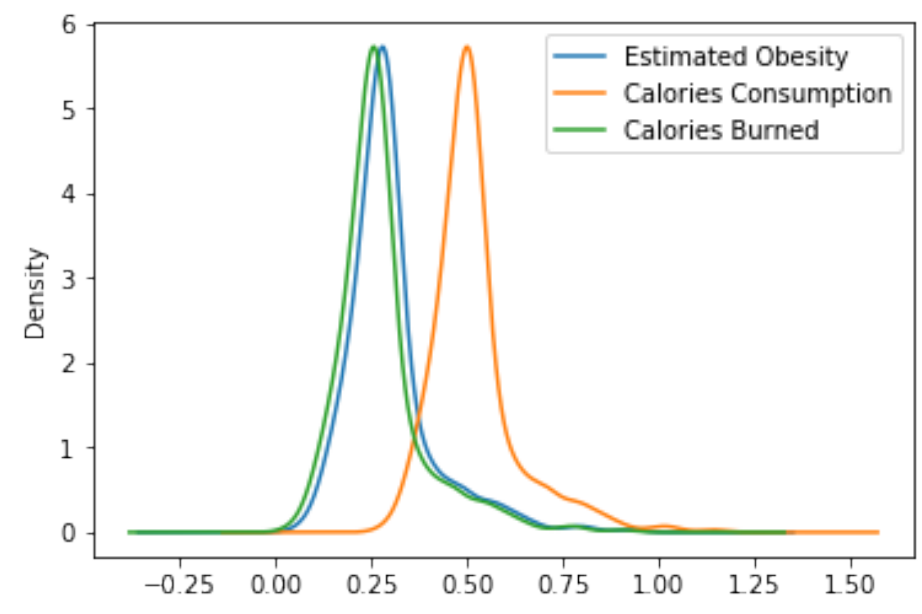

Fig. 5.Density analysis 
Cloud based automated diet recommendation model provides the diet to the patients for protecting the human body from further disease. This model automatically detects the food which is provided to the patients. Various deep learning models are assessed and achieve the reasonable accuracy [14]. The diet monitoring system in zigbee devices is more popular in all the human because of getting instant response. There are some events are generated from the monitoring of the food consumed by the human. Machine learning model with the support of frequency based features with high detection rate [15]. Association rules are used to getting the food preferences of the human and maintain the high level of diet in their regular life. Different categories of the meals are taken and fix the workflow for achieving the high quality of the output with an efficient manner [16]. Music's are classified using automated classification model used to various commercial areas. Machine learning with extreme learning tool provides the high efficient attributes using deep learning method [17]. Proposed density analysis is presented in figure 5. Various algorithms comparison related to the performance is shown in figure 6 . The accuracy of the proposed model is high when compared to other machine learning algorithms.

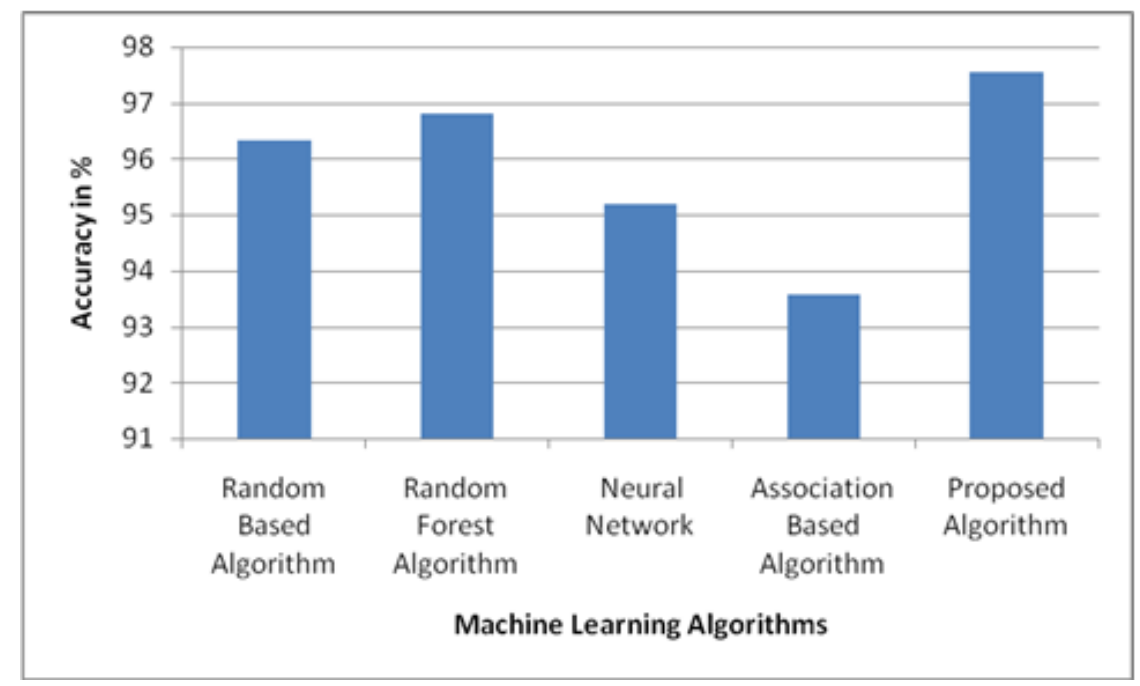

Fig. 6.Performance analysis

\section{Conclusion}

Human heath monitoring system plays vital role in the healthcare industry which needs and provides the support to the life of the human. Traditional methods are using manual intervention leads the accuracy problem, so it will be automated in some level. Automated also provides the support but it is not intelligent still it requires manual intervention. Existing models provides the information to the user based on the present data collected from the human activity. It also suffers the accuracy and reliability problem so it is overcomes by using intelligent with past records. The machine learning models are implemented which achieves the high quality result. The recommendation of the particular information also necessity to the human to select proper food related to their health condition. This model provides the diet 
control report based on the food consumed and burned by the human. Obesity has been estimated which provides the accurate result. The main objective of the proposed algorithm provides the recommendation to the human by taking proper food and prevents the body form disease. In future this model can be applied to the patients of various diseases to consume correct food.

\section{References}

[1] B. Sevilla-Villanueva; K. Gibert; M. Sànchez-Marrè; M. Fitó; M. I. Covas,"Evaluation of Adherence to Nutritional Intervention Through Trajectory Analysis",IEEE Journal of Biomedical and Health Informatics,Volume: 21, Issue: 3, May 2017, Pp.628 - 634.

[2] Senthil Kumar Brindha; NachimuthuSenthil Kumar; Saia Chenkual; S. T. Lalruatfela; Thomas Zomuana; ZothansangaRalte,"Data Mining for Early Gastric Cancer Etiological Factors from DietLifestyle Characteristics",4th International Conference on Intelligent Computing and Control Systems (ICICCS), IEEE, 2020, DOI: 10.1109/ICICCS48265.2020.9121142, Pp.65-73.

[3] Junghyo Lee; PrajwalPaudyal; Ayan Banerjee; Sandeep K. S. Gupta,"FIT-EVE\&ADAM: Estimation of Velocity \& Energy for Automated Diet Activity Monitoring",16th IEEE International Conference on Machine Learning and Applications (ICMLA),DOI: 10.1109/ICMLA.2017.000-7, IEEE, 2017, Pp.1071-1074.

[4] J. Aravind; J. DhaliaSweetlin,"Nutrient facts analysis using supervised learning approaches",Conference on Information and Communication Technology (CICT),IEEE, 2017, DOI: 10.1109/INFOCOMTECH.2017.8340604, Pp. 1-6.

[5] Simone Scardapane; DaniloComminiello; Michele Scarpiniti; Aurelio Uncini, "GP-based kernel evolution for L2-Regularization Networks",IEEE Congress on Evolutionary Computation (CEC), IEEE, 2014, DOI: 10.1109/CEC.2014.6900389, Pp. 1674-1681.

[6] TemiloluwaPrioleau; Elliot Moore; MaysamGhovanloo,"Unobtrusive and Wearable Systems for Automatic Dietary Monitoring",IEEE Transactions on Biomedical Engineering, Volume: 64, Issue: 9, 2017, Pp.2075 - 2089

[7] NunungNurulQomariyah; Ahmad NurulFajar,"Recommender System for e-Learning based on Personal Learning Style",International Seminar on Research of Information Technology and Intelligent Systems (ISRITI), IEEE, 2019, DOI: 10.1109/ISRITI48646.2019.9034568, Pp. 563-567.

[8] Syed Manzar Abbas; KhubaibAmjadAlam,"Exploiting Relevant Context with Soft-Rough Sets in Context-Aware Video Recommender Systems",IEEE International Conference on Fuzzy Systems (FUZZ-IEEE), DOI: 10.1109/FUZZ-IEEE.2019.8859003, 2019, IEEE, Pp. 1-6.

[9] PunamBedi; Sumit Kr. Agarwal,"Managing Security in Aspect Oriented Recommender System",International Conference on Communication Systems and Network Technologies, IEEE, 2011, Pp. 709-713.

[10] Go Hirakawa; Goshi Satoh; Kenji Hisazumi; Yoshitaka Shibata,"Data Gathering System for Recommender System in Tourism",18th International Conference on Network-Based Information Systems, IEEE, 2015, DOI: 10.1109/NBiS.2015.78, PP. 521-525.

[11] Weiwei Yuan; Donghai Guan; LinshanShen; HaiweiPan,"Finding suitable number of recommenders for trust-aware recommender systems: An experimental study",4th IEEE International Conference on Information Science and Technology, IEEE, 2014, DOI: 10.1109/ICIST.2014.6920345, Pp.119-122.

[12] M.P.N.M. Wickramasinghe; D.M. Perera; K.A.D.C.P. Kahandawaarachchi,"Dietary prediction for patients with Chronic Kidney Disease (CKD) by considering blood potassium level using machine learning algorithms", IEEE Life Sciences Conference (LSC), 2017, IEEE, DOI: 10.1109/LSC.2017.8268202, Pp. 300-303.

[13] YingyingZhao,"Research on Entity Recognition in Traditional Chinese Medicine Diet",9th International Conference on Intelligent Human-Machine Systems and Cybernetics (IHMSC), IEEE, 2017, DOI: 10.1109/IHMSC.2017.72, Pp. 282-287.

[14] Celestine Iwendi; Suleman Khan; Joseph Henry Anajemba; Ali Kashif Bashir; FazalNoor,"Realizing an Efficient IoMT-Assisted Patient Diet Recommendation System Through 
Machine Learning Model",Deep Learning Algorithms for Internet of Medical Things,IEEE Access, Volume: 8,Pp. $28462-28474$.

[15] Bo Dong; SubirBiswas, "Wearable diet monitoring through breathing signal analysis",35th Annual International Conference of the IEEE Engineering in Medicine and Biology Society (EMBC), IEEE, 2013, DOI: 10.1109/EMBC.2013.6609718,Pp. 1186-1189.

[16] MohdAnuaruddin Bin Ahmadon; Shingo Yamaguchi, "Meal Preference Extraction and Its Rating Scale for Diet Analysis Using Associative Mining",1st Global Conference on Life Sciences and Technologies (LifeTech), IEEE, 2019, DOI: 10.1109/LifeTech.2019.8884055, Pp. 16-20.

[17] Simone Scardapane; DaniloComminiello; Michele Scarpiniti; Aurelio Uncini,"Music classification using extreme learning machines",8th International Symposium on Image and Signal Processing and Analysis (ISPA), 2013 , IEEE, DOI: 10.1109/ISPA.2013.6703770, pp. 377-381. 